\title{
Review
}

\section{Advances in Studies Related to Interleukin-12 Family and Infectious Diseases}

Bo Li

Tianjin Lung Cancer Institute, Tianjin Medical University General Hospital, Tianjin China

\author{
Keywords \\ Interleukin; IL-12; IL-23; IL-35; Infectious \\ diseases
}

Correspondence

Bo Li,

E-mail: bolitj@126.com

DOI: $10.1515 / i$ ii-2017-0103

\begin{abstract}
Interleukin (IL)-12 family is a group of cytokines composed of heterogeneous molecules and whose members include IL-12, IL-23, IL-27, and IL-35. IL-12 family bridges natural and adaptive immune responses and especially plays a significant role in classical adaptive immune process participated by TH1, TH17, and Treg cells. Members of IL-12 family participate in adaptive immune responses via the Janus kinase-signal transducers and activators of transcription signaling pathway by sharing some subunits and receptors. IL-12 features an extremely complex regulatory network. During resistance of microbial infection, IL-12 and IL-23 mainly show inflammatory effects, whereas IL-27 and IL-35 commonly show antiinflammatory effects. This study reviews advances in studies related to IL-12 family members and infectious diseases and provides references to further reveal functions of IL-12 family members in occurrence and development of infectious diseases.
\end{abstract}

Interleukin (IL)-12 family refers to a group of cytokines composed of heterogeneous molecules, which are similar in structure and covalently bonded. To date, family members include IL-12, IL-23, IL-27, and IL-35. Numerous studies proved that IL-12 and IL-23 induce expression of interferon (IFN) $-\gamma$ and IL-7, respectively, to induce inflammatory effects by regulating Janus kinase-signal transducers and activators of transcription (JAK-STAT) signaling pathway. IL-27 and IL-35 pose antiinflammatory effects by adjusting and controlling the JAK-STAT signaling pathway ${ }^{[1-3]}$. Antiinflammatory effect of IL-27 primarily results from induction of generation of IL-10, whereas antiinflammatory effect of IL-35 is mainly related to induction of generation of IL-35, IL-10, and transforming growth factor- $\beta$ (TGF- $\beta$ ). Increasing number studies considered that IL-12 plays a significant regulatory function in various human diseases, especially during tumor occurrence and development. IL-12 family crucially bridges natural and adaptive immune responses, especially in classical adaptive immunity participated by TH1, TH17, and Treg cell. Members of IL-12 family became important topics in studies and treatment of infectious diseases. This study reviews recent research progress on IL-12 family and infectious diseases to provide references to further reveal function of IL-12 family members in occurrence and development of infectious diseases.

\section{IL-12}

IL-12 was first discovered in 1989 by Trinchieri et al. and named as natural killer (NK) cell stimulatory factor; this molecule is covalently bonded by disulfide bonds of two subunits, namely, P35, Mr 35000 (also named IL-12 $\alpha$ ) and P40, Mr 40000 (also named IL-12 $\beta$ ). In the human body, IL-12 is mainly generated by dendritic cells (DC) and B lymphoblastoid cells stimulated by macrophages and antigens $[4,5]$. IL-12 mediates cytotoxic effects of NK cells by inducing IFN- $\gamma$ expression and promotes mitosis and antiangiogenic effects of T cells. To date, IL-12 is an essential factor in resistance to bacterial and intracellular parasitic infection. This molecule also became the most promising cytokine in treatment of malignant diseases (especially tumors). Studies on mechanism of tumor immune escape considered that IL-12 performs some functions in antitumor angiogenesis activity and reduction of tumor-associated macrophage tumor-supportive activities ${ }^{[6,7]}$. IL-12 also plays an important role in occurrence and development of infectious diseases. During hepatitis $\mathrm{C}$ virus ( $\mathrm{HCV}$ ) infection, IL-12 promotes proliferation and excitation of NK cells and lymphokineactivated killer cell, induces generation of IFN- $\gamma$ and tumor necrosis factor $\alpha$, and enhances clearance of $\mathrm{HCV}$ from natural immunity of hosts ${ }^{[8]}$. Recently, studies of Naderi et al. ${ }^{[9]}$ indicated that IL-12 can be used as gene adjuvant to 
enhance HCV DNA vaccine nonstructural 3, significantly improving immunogenicity of the vaccine. IL-12 can also be used as gene adjuvant of cytomegalovirus DNA vaccine IE 1 to improve immune protection and as molecular adjuvant to stimulate $\mathrm{TH} 1$ immune response and reduce production of IL-4 and IL-10 ${ }^{[10-12]}$. The new heat shock protein 65 DNA combined with IL-12 DNA therapeutic vaccine can effectively prevent pulmonary tuberculosis and features synergistic effects on chemotherapy drugs against Mycobacterium tuberculosis (MTB) ${ }^{[13]}$. In hepatitis B virus (HBV) infection, IL-12 enhances antiviral effect of HBVspecific $\mathrm{CD}^{+} \mathrm{T}$ cells ${ }^{[14]}$. At early stage of influenza virus infection, IL-12 inhibits early viral replication and activation of cytotoxic $\mathrm{T}$ lymphocytes by inducing early expression of IFN- $\gamma$, thereby preventing further development of the disease. Cytokine imbalance caused by continuous HIV-1 infection is an important factor of immunologic defense. High concentration of IL-12 plasmid can effectively reduce HIV-specific humoral immune response by adjusting expression of HIV restriction factor sterile a motif (SAM) and histidine/aspartate (HD) domain-containing protein-1 (type I protein containing SAM and HD structural domains) to resist HIV infection ${ }^{[15]}$. In mice infected with Leishmania parasite, transfected and recombined IL-12 plasmid can eliminate parasitic infection in the body by promoting generation of INF- $\gamma$ by T cells, inhibiting production of IL-4, and enhancing NK activity ${ }^{[16,17]}$.

\section{IL-23}

Through computer prediction, Oppmann et al. ${ }^{[18]}$ discovered that a protein of Mr 19000 can be combined with p35 subunit of IL-12. When Mr 19000 functioned alone, no biological activity was observed. However, when covalently bound to p35 subunit, some biological activity similar to that of IL-12 occurred. Accordingly, IL-23 was discovered. IL-23 is mainly secreted by activated DC and macrophage cells. Similar with IL-12, IL-23 can also induce IFN- $\gamma$ and T cell proliferation. In addition to sharing IL-12R $\beta 1$, IL-12 and IL-23 receptors possess their own unique subunits. IL-12R $\beta 2$, the other receptor of IL-12, is mainly expressed in NK cells and $\gamma \delta \mathrm{T}$ cells. IL-23R is restrictedly expressed in T cell subsets, a few B cells, and lymphocytes ${ }^{[19]}$. As inflammatory cell, IL-23 acts on memory $\mathrm{T}$ cells, affecting immune, potential antitumor, and antiinfection responses. IL-23 can effectively stimulate differentiation and excitation of TH17 cell subset ${ }^{[20]}$. TH17 cells refer to $\mathrm{CD}^{+}{ }^{+} \mathrm{T}^{4}$ cell subsets of specific secretory IL-17.
In addition to IL-17, IL-21 and IL-22 are the major cytokines secreted by TH17 cells ${ }^{[21]}$, which play an important role in pathogenesis of inflammatory diseases, such as experimental autoimmune encephalomyelitis, rheumatoid arthritis, and colitis. In recent years, increasing studies showed that IL-23 plays an important role in resistance to bacteria, parasites, viruses, and fungal infection. Kagami et al. ${ }^{[22]}$ illustrated that IL-23 is one of the indispensable factors determining immune clearance of Candida albicans infection. Verreck et al. observed dual function of macrophages after MTB infection. In the presence of granulocyte macrophage colonystimulating factor, type I macrophages secrete high levels of IL-23 but not IL-12 and promote cellular immune function of organisms and secretion of IL-12 via IFN- $\gamma$ as a second messenger. However, type II macrophages can secrete IL-10 to reduce cellular immune function of the body, indicating that IL-23 plays an important role in antituberculous immune response participated by macrophages ${ }^{[23]}$. In the aspect of antiparasitic infection, IL-23 P19 subunit knockout mice were highly susceptible to infection with Pneumocystis jirovecii, and time of removal of bacteria in the body was significantly prolonged ${ }^{[24]}$. IL-23 was also indispensable in immune process of antituberculosis, antiviral, and antiparasitic infection, which directly involve IL-17 and TH-17. Palma et al. ${ }^{[25]}$ suggested that MTB lipoprotein PstS1 stimulates DC to produce IL-23, induces CD4 ${ }^{+} \mathrm{T}$ cells to secrete IFN- $\gamma$, IL-17, and IL-22, and participates in antituberculotic immune process directly mediated by IL-17 and TH17. Wang et al. ${ }^{[26]}$ discovered that high expression of $\mathrm{T}$ cell immunoglobulin mucin domain-3 protein in monocytes of chronic HCV-infected patients with failed $\mathrm{HBV}$ vaccine immunity can regulate generation of IL-12 and IL-23 and mediate IL-17 and THL17 to participate in antiviral immune process. Ishida et al. ${ }^{[27]}$ indicated that in mice infected with Plasmodium, IL-23 is necessary for secretion of IL-17 by mouse $\mathrm{CD} 4^{+} \mathrm{T}$ cells to resist infection. IL-23 knockout mice exhibited low IL-17 level, high parasitemia, and early death.

\section{IL-27}

IL-27 comprises EBI3 and p28 subunits. EBI3 and p28 subunits were discovered in 1996 and 1998, respectively. In 2001, Pflanz et al. elucidated IL-27 by biochemical methods, and its receptors were identified in $2004^{[28]}$. IL-27 is mainly generated by antigen-presenting cells, such as activated monocytes and DC and their receptors, IL-27R (also named 
WSX-1) and gp130, and mainly expressed in the thymus, spleen, and peripheral blood lymphocytes, especially on surfaces of $\mathrm{CD}^{+}{ }^{+} \mathrm{T}$ cells and NK cells ${ }^{[28,29]}$. Combination of IL-27 and its receptors can activate spleen tyrosine kinaseSTAT signaling pathway, upregulate expression of T-bet transcription factor, induce $\mathrm{CD} 4^{+} \mathrm{T}$ cells to differentiate into TH1 cells, and collaborate with IL-12 to promote initial $\mathrm{T}$ cells to produce IFN- $\gamma$. During immune response to Plasmodium falciparum infection, IL-27 regulates TH1 cells through its receptor IL-27R pathway to achieve clearance of pathogens ${ }^{[29]}$. In the course of acute HBV infection, IL-27 promotes elimination of the virus by promoting immune response of TH1. However, in chronic hepatitis B patients, IL-27 enhances inflammatory response of the liver by promoting virus clearance, which aggravates hepatocyte injury participated by TH17 cells ${ }^{[30]}$. Recently, Cao ${ }^{[31]}$ observed high expression of IL-27 in sputum and serum of patients with chronic obstructive pulmonary disease and pulmonary tuberculosis; these conditions can induce high expression of CXCL10 in airway epithelial cells. IL-27 inhibits HIV-1 replication in CD4 $4^{+} \mathrm{T}$ cells and macrophages in an IFN- $\gamma$-dependent manner and is assumed an effective inhibitor of HIV-1 infected cells ${ }^{[32]}$.

\section{IL-35}

In 2007, Collison et al. discovered that EB13 and p35 subunits were highly expressed in Foxp3-Treg cells of mice while not expressed in resting or activated $\mathrm{CD} 4^{+} \mathrm{T}$ cells. The following studies showed that both antigens stimulate human Treg, and nonstimulated mouse Treg can secrete 35, whereas non-antigen stimulated human Treg cannot secrete IL-35 [33,34]. Toll-like receptor 4 and CD40-activated B cells can also secrete IL-35 ${ }^{[35]}$. IL-35 is a negative immune regulatory factor playing an important role in inhibiting proliferation of effector T cells, TH17 cell differentiation, and IL-17 composition. Treg-derived IL-35 can induce transformation of $\mathrm{CD}^{+}{ }^{+}$effector $\mathrm{T}$ cells into Treg subsets of specifically secreted IL-35 cells, that is, iTr35 cells, with inhibitory functions ${ }^{[36]}$. ITr35 cells do not express Foxp3, TGF- $\beta$, and IL-10. Studies of adoption of iTr35 cells in autoimmune disease and inflammatory animal models of five different bodies showed that $i \mathrm{Tr} 35$ cells posed protective effects on these disease models ${ }^{[36]}$. Human Treg can also express IL-35 and induce generation of $i \operatorname{Tr} 35$ cells in IL-35-dependent manner ${ }^{[34]}$. In recent years, most studies confirmed that IL-35 is highly expressed in tumor microenvironment and may also exist in immune escape jointly participated by tumor-derived IL-35 ${ }^{[37,38]}$. IL-35 is highly expressed in colon cancer and is positively correlated with tumor malignancy [39]. Further studies should still determine whether Tregderived IL-35 mediates immune tolerance. Xie et al. ${ }^{[40]}$ noted proinflammatory effects of IL-35 on pathogenesis of viral myocarditis, and overexpression of IL-35 exhibited protective effects against viral myocarditis. Recently, Shen et al. ${ }^{[35]}$ observed deficiency in recovery ability of experimental allergic encephalomyelitis (EAE) medicated by $\mathrm{T}$ cell of IL-35 subunit (EB13 or p35) of B cell gene knockout mice but extremely strong ability for anti-Salmonella infection. This finding indicates that secretion of IL-35 by B cells plays crucial immunomodulatory effects in EAE and Salmonella infection and is a potential target for treatment of autoimmune and infectious diseases.

\section{Prospective}

In recent years, in-depth studies affirmed status of IL-12 family members in infectious diseases. To date, as target for treatment of infectious diseases, IL-12 family members became an important topic in most scientific research. However, with similarity and diversity in molecular structure, receptor structure, and function of IL-12 family members, progress in related studies on clinical application is still relatively slow. To determine the balance point of IL-12 family members during microbial infection resistance, all challenges encountered by IL-12 family members during application in disease diagnosis, treatment, and prognosis improvement must be addressed; these issues include designing rational drugs to regulate IL-12 family members and clarifying roles of IL-12 family members in occurrence and development of different infectious diseases.

\section{Declarations}

\section{Acknowledgements}

No.

\section{Competing interests}

The author declare that she has no competing interest.

\section{Authors' contributions}

B Li made the literature analysis and wrote, discussed and revised the manuscript of this review. 


\section{References}

1 Kalliolias GD1, Ivashkiv LB. IL-27 activates human monocytes via STAT1 and suppresses IL-10 production but the inflammatory functions of IL-27 are abrogated by TLRs and p38. J Immunol, 2008, 180(9): 6325-6333. infection, inflammation and autoimmune disorders. Inflamm Allergy Drug Targets, 2009,8(1):40-52.

3 Shimizu J,Kaneko F,Suzuki N. Skewed helper T-cell responses to IL-12 family cytokines produced by antigen-presenting cells and the genetic background in Behcet's disease. Genet Res Int, 2013, 2013:363859.

4 Yuzhalin AE1, Kutikhin AG. Interleukin-12: clinical usage and molecular markers of cancer susceptibility. Growth Factors, 2012, 30(3):176-191.

5 Friedmann E1, Hauben E, Maylandt K, et al. SPPL2a and SPPL2b promote intramembrane proteolysis of TNFa in activated dendritic cells to trigger IL-12 production. Nat Cell Biol, 2006,8 (8): 843-848.

6 Banerjee S, Halder K, Bose A, et al. TLR signaling-mediated differential histone modification at IL-10 and IL-12 promoter region leads to functional impairments in tumor-associated macrophages. Carcinogenesis, 2012, 32(12): 1789-1797.

7 Ko SY, Ladanyi A, Lengyel E, et al. Expression of the homeobox gene HOXA9 in ovarian cancer induces peritoneal macrophages to acquire an M2 tumor-promoting phenotype. Am J Pathol, 2014, 184(1):271-281

8 Clerici M, Saresella M, Trabattoni D. Single-cell analysis of cytokine production shows different immune profiles in multiple sclerosis patients with active or quiescent disease. J Neuroimmunol, 2001, 121(1-2):88-101.

9 Naderi M, Saeedi A, Moradi A, et al. Interleukin-12 as a genetic adjuvant enhances hepatitis C virus NS3 DNA vaccine immunogenicity. Virol Sin, 2013,28(3): 167-173.

10 Metzger DW. Interleukin-12 as an adjuvant for induction of protective antibody responses. Cytokine, 2010, 52(1-2):102-107.

11 Wei F, Wang $\mathrm{H}$, Zhang J, et al. Pharmacokinetics of combined gene therapy expressing constitutive human GM-CSF and hyperthermiaregulated human IL-12. J Exp Clin Cancer Res, 2013,32:5.

12 Saghafian-Hedengren S, Sohlberg E, Theorell J, et al. Epstein-Barr virus coinfection in children boosts cytomegalovirus-induced differentiation of natural killer cells. J Virol, 2013,87 (24):13446-13455.

13 Kita Y, Hashimoto S, Nakajima T, et al. Novel therapeutic vaccines[(HSP65 + IL-12)DNA-, granulysin-and Ksp37-vaccine] against tuberculosis and synergistic effects in the combination with chemotherapy. Hum Vaccin Immunother, 2012,9(3):526-533.

14 Schurich A, Pallett LJ, Lubowiecki M, et al. The third signal cytokine IL-12 rescues the anti-viral function of exhausted HBV-specific CD8 T cells. PLoS Pathog, 2013,9(3): e1003208.

15 Pauls E, Jimenez E, Ruiz A, et al. Restriction of HIV-1 replication in primary macrophages by IL-12 and IL-18 through the upregulation of SAMHD1. J Immunol, 2013,190(9): 4736-4741.

16 Ricardo-Carter C, Favila M, Polando RE, et al. Leishmania major inhibits
IL-12 in macrophages by signalling through CR3 ( CD11b /CD18) and down-regulation of ETS-mediated transcription. Parasite Immunol, 2013,35(12):409-420

17 Hugentobler F, Yam KK, Gillard J, et al. Immunization against Leishmania major infection using LACK-and IL-12-expressing Lactococcus lactis induces delay in footpad swelling. PLoS One, 2012,7(2):e30945.

18 Oppmann B, Lesley R, Blom B, et al. Novel p19 protein engages IL-12p40 to form a cytokine,IL-23, with biological activities similar as well as distinct from IL-12. Immunity, 2000,13 (5):715-725.

19 Chognard G, Bellemare L, Pelletier AN, et al. The dichotomous pattern of IL-12R and IL-23R expression elucidates the role of IL-12 and IL-23 in inflammation. PLoS One, 2014,9(2):e89092.

20 Boniface K, Blom B, Liu YJ, et al. From interleukin-23 to T-helper 17 cells: human T-helper cell differentiation revisited. Immunol Rev, 2008,226:132-146.

21 De Simone V, Pallone F, Monteleone G, et al. Role of TH17 cytokines in the control of colorectal cancer. Oncoimmunology, 2013,2(12): e26617.

22 Kagami S, Rizzo HL, Kurtz SE, et al. IL-23 and IL-17A,but not IL-12 and IL-22, are required for optimal skin host defense against Candida albicans. J Immunol, 2010, 185(9):5453-5462.

23 Verreck FA, de Boer T, Langenberg DM. Human IL-23-producing type 1 macrophages promote but IL-10-producing type 2 macrophages subvert immunity to(myco) bacteria. PNAS, 2004,101(13): 4560-4565.

24 Rudner XL, Happel KI, Young EA, et al. Interleukin-23(IL-23)-IL-17 cytokine axis in murine Pneumocystis carinii infection. Infect Immun, 2007,75(6):3055-3061.

25 Palma C, Schiavoni G, Abalsamo L, et al. Mycobacterium tuberculosis PstS1 amplifies IFN- $\gamma$ and induces IL-17 /IL-22 responses by unrelated memory CD $4+\mathrm{T}$ cells via dendritic cell activation. Eur J Immunol, 2013,43(9):2386-2397.

26 Wang JM, Ma CJ, Li GY, et al. Tim-3 alters the balance of IL-12 /IL-23 and drives TH17 cells: role in hepatitis B vaccine failure during hepatitis C infection. Vaccine, 2013,31(18):2238-2245.

27 Ishida H, Imai T, Suzue K, et al. IL-23 protection against Plasmodium berghei infection in mice is partially dependent on IL-17 from macrophages. Eur J Immunol, 2013,43(10):2696-2706.

28 Pflanz S,Hibbert L,Mattson J,et al. WSX-1 and glycoprotein 130 constitute a signal-transducing receptor for IL-27. J Immunol, 2004,172(4): 2225-2231.

29 Villegas-Mendez A, de Souza JB, Lavelle SW, et al. IL-27 receptor signalling restricts the formation of pathogenic, terminally differentiated TH1 cells during malaria infection by repressing IL-12 dependent signals. PLoS Pathog, 2013,9(4):e1003293.

30 Zhang GL, Xie DY, Ye YN, et al. High level of IL-27 positively correlated with TH17 cells may indicate liver injury in patients infected with HBV. Liver Int, 2014,34(2):266-273.

31 Cao J, Zhang L, Li D, et al. IL-27 is elevated in patients with COPD and patients with pulmonary TB and induces human bronchial epithelial 
cells to produce CXCL10. Chest, 2012,141 (1 ): 121-130.

32 Chen Q, Swaminathan S, Yang D, et al. Interleukin-27 is a potent inhibitor of cis HIV-1 replication in monocyte-derived dendritic cells via a type I interferon-independent pathway. PLoS One, 2013, 8(3):e59194.

33 Collison LW, Workman CJ, Kuo TT, et al. The inhibitory cytokine IL-35 contributes to regulatory T-cell function. Nature, 2007, 450(7169):566-569.

34 Bardel E, Larousserie F, Charlot-Rabiega P, et al. Human CD4 +CD25 + Foxp3 + regulatory T cells do not constitutively express IL-35. J Immunol, 2008,181(10):6898-6905.

35 Shen P, Roch T, Lampropoulou V, et al. IL-35-producing B cells are critical regulators of immunity during autoimmune and infectious diseases. Nature, 2014,doi: 10. 1038 /nature12979.

36 Collison LW, Chaturvedi V, Henderson AL, et al. IL-35-mediated induction of a potent regulatory $\mathrm{T}$ cell population. Nat Immunol, 2010,11(12):1093-1101.

37 Long J, Zhang X, Wen M, et al. IL-35 over-expression increases apoptosis sensitivity and suppresses cell growth in human cancer cells. Biochem Biophys Res Commun, 2013,430(1):364-369.

38 Wang Z, Liu JQ, Liu Z. Tumor-derived IL-35 promotes tumor growth by enhancing myeloid cell accumulation and angiogenesis. J Immunol, 2013,190(5): 2415-2423.

39 Zeng JC, Zhang Z, Li TY, et al. Assessing the role of IL-35 in colorectal cancer progression and prognosis. Int J Clin Exp Pathol, 2013,6(9):1806-1816.

40 Xie Y, Chen R, Zhang X, et al. The role of TH17 cells and regulatory $\mathrm{T}$ cells in Coxsackievirus B3-induced myocarditis. Virology, 2011, 421(1):78-84. 